\title{
Prognostic Benefit of 3D and 4D Ultrasound for Fetal Airway Assessment and Implications for Delivery
}

\author{
Megan Gutierrez ${ }^{1,2 *}$, Meagan CampolHaynes ${ }^{3}$, Megan Guiterrez ${ }^{1}$, Marcos Cordoba Munoz ${ }^{4}$, Ana Monteagudo ${ }^{5,6,7}$ \\ and Ilan Timor-Tritsch ${ }^{6}$
}

${ }^{1}$ Department of OBGYN Division of Maternal Fetal Medicine Livingston, Saint Barnabas Medical Center, NJ

${ }^{2}$ Perinatal Associates Livingston, USA

${ }^{3}$ Yale University School of Medicine New Haven, USA

4Department of OBGYN Division of Maternal Fetal Medicine Ann Arbor, Michigan State University, USA

5Maternal Fetal Medicine Associates New York, USA

6Department of OBGYN Division of Maternal Fetal Medicine, New York University, USA

7Department of OBGYN Division of Maternal Fetal Medicine, Mt. Sinai School of New York, USA

Submission: October 10, 2018 ; Published: November 21, 2018

*Corresponding author: Margaret Dziadosz MD, New Jersey Perinatal Associates, 94 Old Short Hills Road East Wing Suite 402 Livingston, NJ 0039, USA

\begin{abstract}
Background: Fetal craniofacial anomalies carry varied probability of neonatal airway obstruction. The Ex Utero Intrapartum Treatment (EXIT) procedure can secure the airway at birth, however carries significant maternal risks. 3D ultrasound may predict degree of airway obstruction prior to delivery.

Case: We present four cases of fetal nasopharyngeal or craniofacial abnormalities concerning for airway compromise. Diagnoses included epulis, congenital hemangioma, tetralogy of Fallot, and Treacher Collins syndrome. Concrete ultrasonographic findings of fetal swallowing, including [1], normal amniotic fluid volume, [2], presence of a stomach bubble [3], uninterrupted bidirectional Doppler flow of amniotic fluid, and (4) fluid in the trachea, indicated patent fetal airway [1-3].

Conclusion: These four ultrasonographic signs indicate that the fetal airway is unobstructed. Antenatal delivery planning, including necessity for EXIT, is greatly improved using 3D ultrasound.

Keywords: 3D Ultrasound; 4D Ultrasound; Fetal airway; Neonatal airway; EXIT; Ultra sonographic markers

Abbreviatations: 3DUS: Three-Dimensional Ultrasound; EXIT: Ex Utero Intrapartum Treatment; AFV: Amniotic Fluid Volume; SB: Stomach Bubble; RICH: Rapidly Involuting Congenital Hemangioma; US: Ultrasound; TUI: Tomographic Imaging
\end{abstract}

\section{Introduction}

Advances in ultrasound technology have transformed our abilities in prenatal diagnosis. Specifically, the value of three-dimensional ultrasound (3DUS) has profound clinical implications when diagnosing fetal craniofacial and nasopharyngeal anomalies with the potential for airway obstruction [1,2]. Varying diagnoses carry different probabilities of neonatal breathing impairment, difficult intubation, asphyxia or neonatal death. In complement to 2D ultrasound, both 3D and 4D ultrasound (4DUS) technologies promote clinically relevant, enhanced analysis of complex fetal anatomy. A thorough assessment using 3D and 4DUSprovidescrucial prognostic information regarding the need for special delivery methods, ie. Ex Utero Intrapartum Treatment (EXIT) procedure, a technique that allows for prolonged maintenance of uteroplacental circulation for fetal interventions at time of cesarean section. Importantly, EXIT introduces significant maternal risks including aspiration, hemorrhage, hypoxia, and the risks of general anesthesia.

\section{Methods}

In this article, we present four cases high lighting utility of3DUS and 4DUSin delivery planning and propose sonographic markers for airway patency. In all cases, successful prediction of fetal airway status allowed avoidance of potentially morbid 
interventions like EXIT. Through careful assessment of fetal airway, both neonatal and maternal morbidity and mortality may be decreased and safe delivery promoted.

\section{Results}

\section{Case \#1: Oral Mass}

A 34-year-old, para 1001, was referred for evaluation after ultrasound (US) at 28 weeks identified an oral mass on a female fetus. On 3DUS, the exophytic oral mass measured $2 \times 1.8 \mathrm{~cm}$, was solid and homogeneous in texture with limited vascularity, protruded from the mandible, and was mobile. Serial 3DUS and 4D HD live technology revealed uninterrupted, bidirectional Doppler flow of amniotic fluid through the nares and mouth, a normalized amniotic fluid volume (AFV), and a stomach bubble (SB) (Figure 1). The patient underwent cesarean delivery under regional anesthesia at 37 weeks per multidisciplinary decision. Immediate vigorous fetal cry occurred without breathing compromise. Fiber optic endotracheal evaluation was performed without evidence of airway obstruction. Final pathology of excised mass revealed Epulis $(3,4)$.

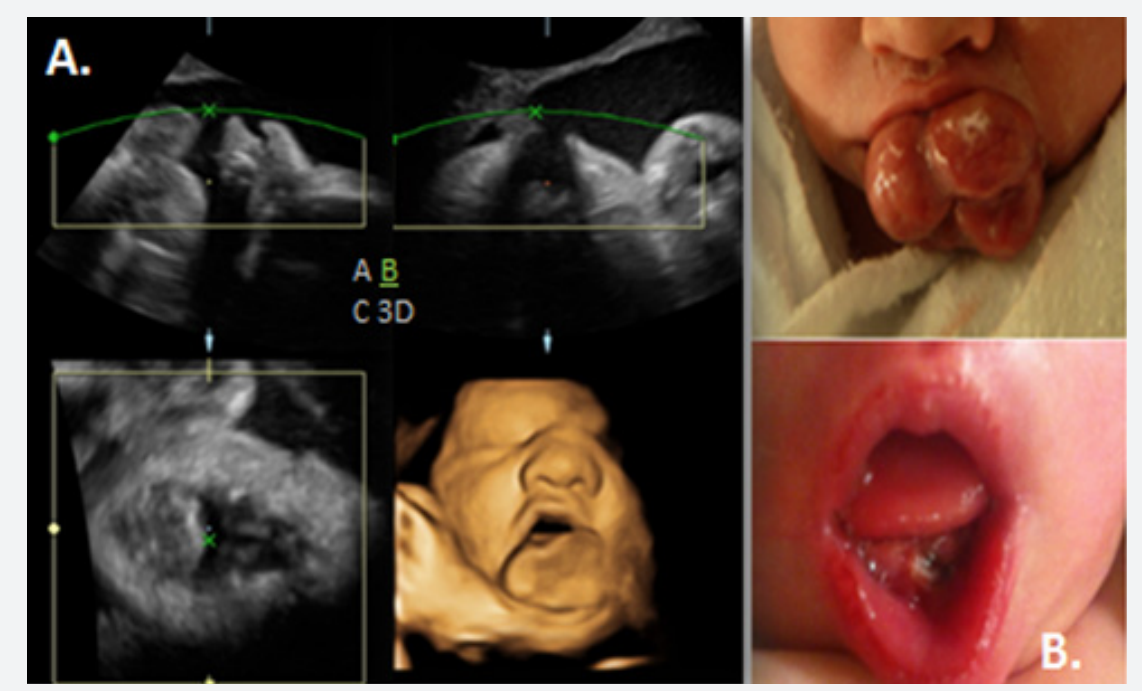

Figure 1: Epulis(A)A three-dimensional surface showing the fetal face with the protruding oral mass and the fetal nares that appear not obstructed by the oral mass. (B)Photographs documenting the postnatal appearance of the tumor before and after excision.

\section{Case \#2: Neck Mass}

A 32-year-old, para 0010, was evaluated at 32 weeks when US revealed an incidental mass of the neck. 3DUS revealed a $3.7 \times 2.8 \times 2.6 \mathrm{~cm}$ homogeneous, superficial, mobile, well circumscribed, left sided mandibular mass with peripheral blood flow and scant internal blood flow. Fetal swallowing and breathing were noted via 4D HD live imaging, fluid was seen within the trachea via 3D rendering manipulation and tomography, there was normal AFV, and a SB was present (Figure 2) [5]. The mass remained stable in size. Cesarean delivery was scheduled at 37 weeks under regional anesthesia with a multidisciplinary team. The infant exhibited immediate spontaneous cry without breathing difficulty. On gross examination, the pedunculated cervical neck mass was soft with violaceous to blue color centrally, without ulceration or hemorrhage. Diagnosis of Rapidly Involuting Congenital Hemangioma (RICH) was made and expectant management planned.

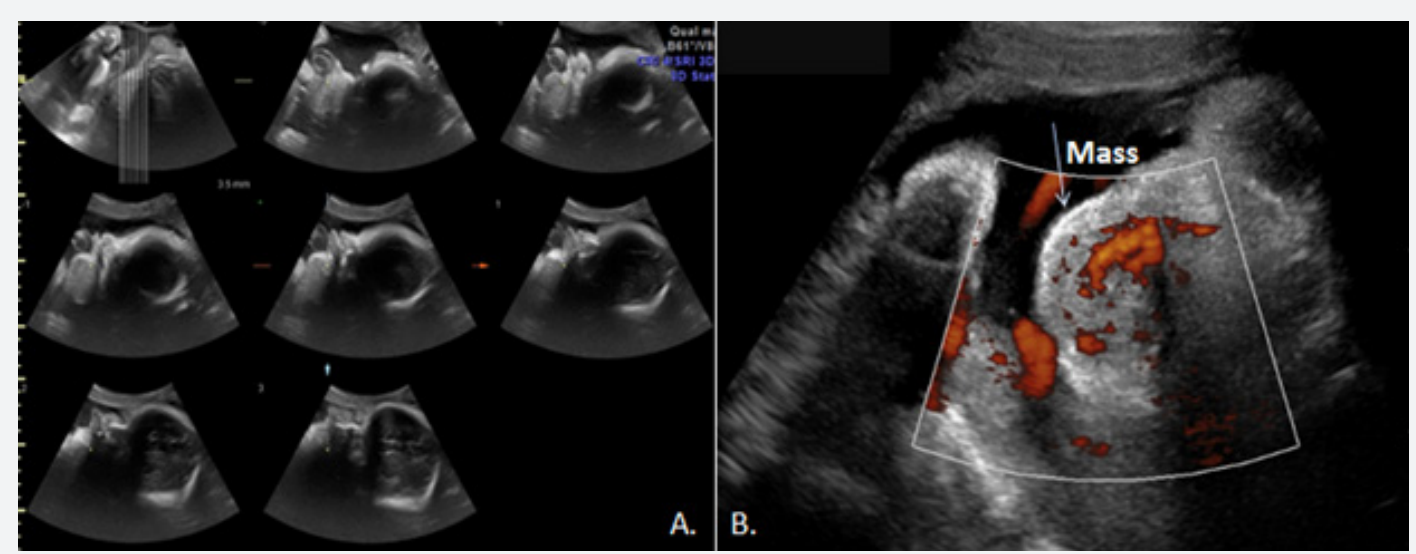

Figure 2: Hemangioma(A)TUI shows the fetal neck mass (B)Axial Doppler sonogram of the fetal neck reveals isoechoic homogeneous subcutaneous mass with increased vascularity. 


\section{Case \#3: Mediastinal mass effect}

A 37-year-old, para 1011, was evaluated at 27 weeks for fetal cardiac anomaly. The gravid a had a significant medical history of congenital pulmonary stenosis and severe right ventricular hypertrophy. Fetal echocardiogram revealed tetralogy of Fallot with severe right ventricular outflow tract stenosis, ventricular septal defect, congenital absence of the pulmonary valve and aneurysmal dilation of the pulmonary artery, dilation and hypertrophy of the right ventricle, and resultant compression of fetal trachea. Evaluation of the fetal oropharynx using 3DUSand 4D HD Live revealed bidirectional flow with breathing and swallowing, a clear tracheal passage, a SB, and normal AFV (Figure 3). A repeat cesarean was performed at 36 weeks due to deterioration of maternal cardiac status under general anesthesia with a multidisciplinary team. Spontaneous cry was noted upon delivery. The infant underwent cardiac repair on day of life 7.

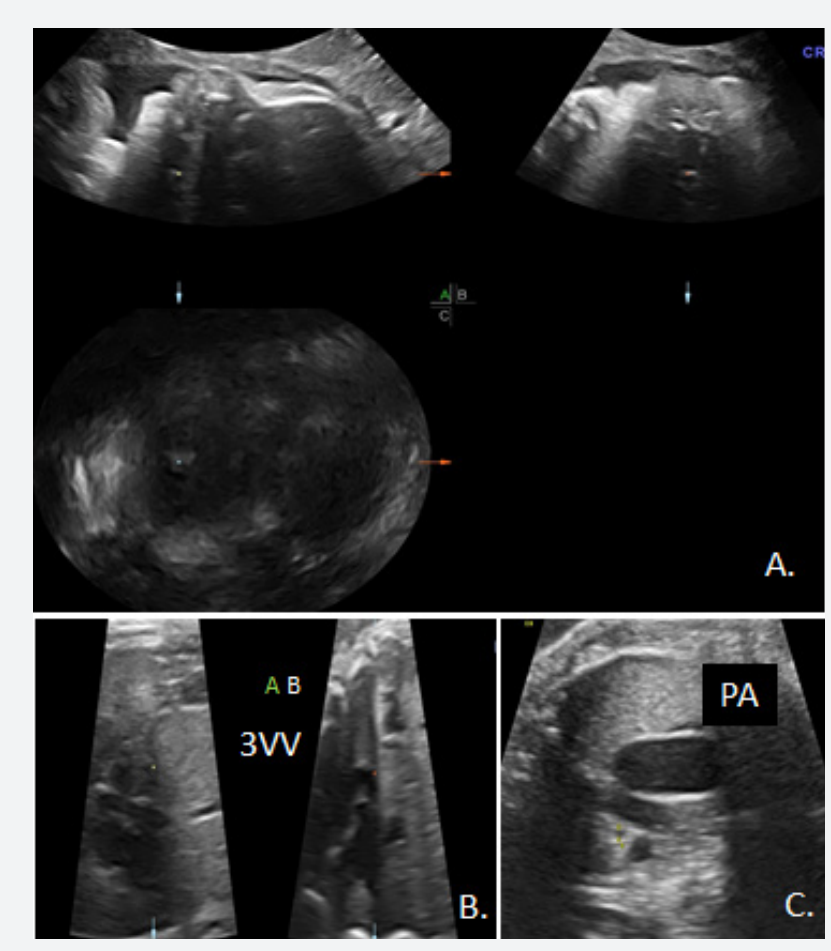

Figure 3: Tetralogy of Fallot(A)3D surface rendering of fetal airway and upper thoracic cavity (B)Multiplanar view of compressed trachea in the three-vessel view (3VV) (C) 2D US view of dilated pulmonary artery (PA).

\section{Case \#4: Micrognathia}

A 21-year-old, para 2001, presented for prenatal care at 18 weeks with her male partner, who has Treacher Collin's syndrome. Serial 3DUS and 4DHDlive US revealed a fetal unilateral ear bud, recessed mandible, and underdeveloped zygomatic arches. The fetal SB and AFV remained normal. 3D rendering allowed accurate depiction of the fetal face and mandibular measures (Figure 4). At 39 weeks repeat cesarean under regional anesthesia was performed with multidisciplinary teams. Immediate spontaneous cry was noted.
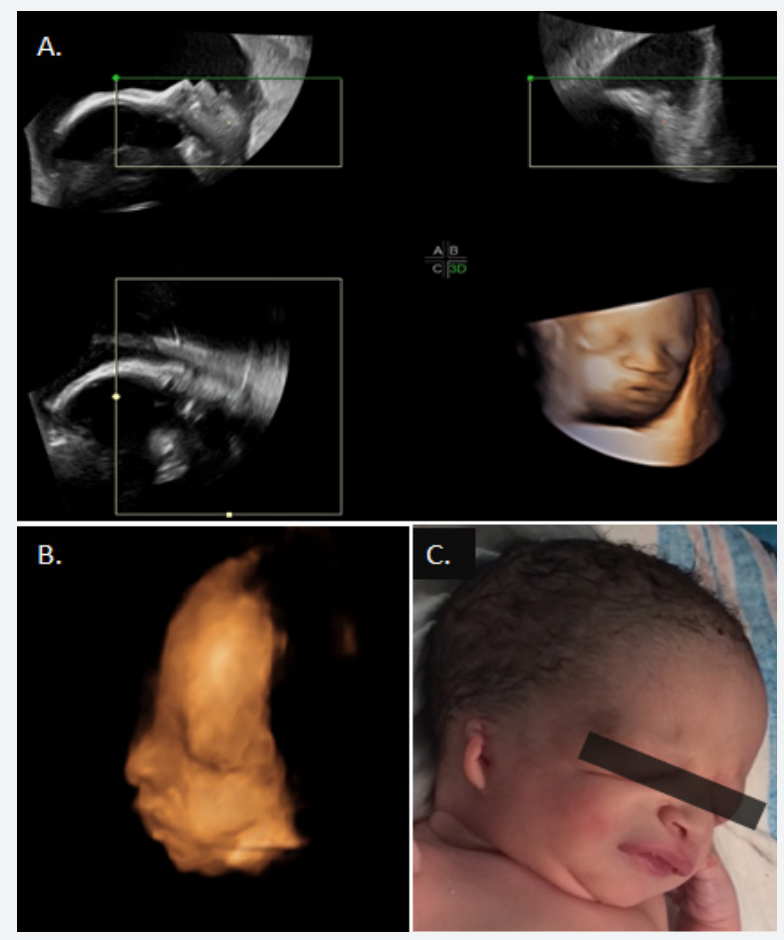

Figure 4: Treacher Collin's syndrome (A) Three-dimensional image of the fetal face.(B) Three-dimensional image of the face in right lateral view. Micrognathia was clearly depicted. (C) Image of the newborn face just after delivery.

\section{Discussion}

Advancing technologies in ultrasound are vital for evaluation of complex prenatal anatomy $[2,5,6]$. For fetal anomalies with potential respiratory compromise and difficult intubation at birth, it may be the primary decision making tool in safe delivery planning. Through a comprehensive evaluation of the amniotic fluid volume, stomach bubble, oral and nasal passages, and nasopharynx- trachea using 3DUS and 4DUS, patency and pliability of the airway can be accurately predicted.

Oral tumors and craniofacial malformations may cause obstruction of the fetal airway at time of birth, leading to potentially life-threatening respiratory distress. When fetal airway access is compromised, one technique for optimizing fetal oxygen status at birth is the Ex Utero Intrapartum Treatment procedure, or EXIT. EXIT allows for prolonged maintenance of utero-placental circulation for fetal interventions at time of cesarean section through partial fetal delivery with continued placental flow. Once a fetal airway is secured, the infant may be fully delivered [3].

However, EXIT introduces serious maternal risks. Aspiration pneumonitis is common as the lower esophageal sphincter pressure is reduced with increased compression by the gravid uterus on the stomach. Combining this natural physiology with increased gastric acid production poses a danger during general anesthesia. Additionally, due to the decreased preload during supine positioning, the compromised cardiovascular system 
may experience hypotension with decreased uterine artery perfusion. Lastly, the prolonged length of EXIT leads to increased blood loss during cesarean delivery [3]. While EXIT is a valuable tool, its significant complications dictate that it should only be used if necessary.

Through detailed views of fetal anatomy, 3DUS helped to predict airway patency in each case. While EXIT was considered for all due to concern for neonatal breathing impairment and difficult intubation, antenatal decision was made against EXIT due to reassuring US signs of patent airway. Multidisciplinary planning between obstetrical, anesthesia, pediatric ENT, and NICU teams concurred that fetal outcome would not benefit via EXIT while increasing unnecessary risks for the mother. However, multidisciplinary teams were present at delivery for precaution. Ultimately, all neonates projected vigorous, immediate cries upon delivery without obstructed breathing and did not require intubation.

Compared to 2D ultrasonography, 3DUS has proven advantageous in the detection of abnormal fetal anatomy and airway obstruction. It is not only useful in predicting the severity of a defect, but also in providing more convincing evidence of a normal fetus in cases at increased risk of malformation [6]. As an adjunct2D ultrasonography, 3DUS adds value in diagnosing various fetal head and neck abnormalities, including micrognathia and other profile malformations, cleft lip and palate, metopic suture abnormalities, nasal bone, ear or orbit abnormalities, neural tube defects, and skeletal malformations [7].

In each case, various 3D technologies were applied for a complete anatomical assessment. 3D US surface rendering allows for accurate depiction of the fetal face and profile. The electronic scalpel assists in removal of obstructing adjacent structures such as placenta or cord that obscure the area of interest. 3D volume acquisition and tomographic imaging (TUI) enable multi-slice analysis that extends MRI capability with volume rendering and plane manipulation. Multiplanar display allows images to be displayed at right angles to each other in sagittal, coronal, or axial planes. By choosing a single point of intersection and traversing the three planes, one facilitates an improved spatial relationship of complex anatomical defects [1,2,5-8]. HD live imaging enables 3DUS reconstruction at a frame rate of up to 20 images per second, yielding a smooth and dynamic real time 3D image. With the addition of Doppler, it is possible to visualize real-time active streaming of unimpaired bidirectional fluid flow in the fetal body.

In addition to diagnostic purposes, 3DUS is a powerful tool for antenatal communication for both patients and providers [6]. Through a realistic pictorial representation, it helps to improve understanding regarding the defect as well as necessity for prenatal genetic testing or procedures, mode of delivery, prognosis, and neonatal expectations. With increased understanding among families and physicians, coordination of care and treatment planning including interventions in neonatology, pediatric ENT, pediatric surgery, and plastic surgery can be expedited.

We propose specific sonographic findings paramount in evaluating fetal airway patency:

a) Normal amniotic fluid volume index,

b) Presence of a stomach bubble,

c) Uninterrupted bidirectional Doppler flow of amniotic fluid through the nasopharynx and oral cavity, and

d) Fluid in the trachea.

These markers demonstrate normal fetal swallowing and breathing and indicate that EXIT is unnecessary, although it is prudent to have multidisciplinary teams at delivery prepared to address unanticipated events. With adequate information about airway patency, a potentially morbid procedure may be avoided. As we have highlighted through this case series of fetal diagnoses with risk for airway compromise, there is a definitive predictive role for 3DUS in obstetrical delivery planning. Concrete sonographic markers may be used when fetal airway anomaly is detected and directed obstetrical delivery preparation is warranted. The ability to render and manipulate images through 3DUS in order to thoroughly evaluate the patency of the fetal oral cavity and airway is paramount in directing both fetal and maternal care and ensures the least overall morbidity.

\section{References}

1. Duckelmann AM, Kalache KD (2010) Three-dimensional ultrasound in evaluating the fetus. Prenat Diagn 30(7): 631-638.

2. Timor-Tritsch IE, Monteagudo A (2007) Three and four-dimensional ultrasound in obstetrics and gynecology. Curr Opin Obstet Gynecol 19(2): 157-175.

3. Laje P, Howell LJ, Johnson MP, Hedrick HL, Flake AW, et al, (2013) Perinatal management of congenital oropharyngeal tumors: the ex utero intrapartum treatment (EXIT) approach. J Pediatr Surg 48(10): 2005-2010.

4. Petermann L, et al. (2014) Relevance of fetal 2D/3D ultrasonography for assessing potential airway obstruction in congenital epulis. Ultrasound ObstetGynecol.

5. Yagel S, Cohen SM, Messing B, Valsky DV (2009) Three-dimensional and four-dimensional ultrasound applications in fetal medicine. Curr Opin Obstet Gynecol 21(2): 167-174.

6. Merz E, Abramovicz J, Baba K, Blaas HG, Deng J, et al. (2012) 3D imaging of the fetal face - recommendations from the International 3D Focus Group. Ultraschall Med 33(2): 175-182.

7. Gonçalves LF, Lee W, Espinoza J, Romero R (2005) Three- and 4-dimensional ultrasound in obstetric practice: does it help? J Ultrasound Med 24(12): 1599-1624.

8. Benacerraf BR, Shipp TD, Bromley B (2006) Three-dimensional US of the fetus: volume imaging. Radiology 238(3): 988-996. 
(C) This work is licensed under Creative CC (i) Commons Attribution 4.0 License DOI: 10.19080/JGWH.2018.12.555848

\section{Your next submission with Juniper Publishers will reach you the below assets}

- Quality Editorial service

- Swift Peer Review

- Reprints availability

- E-prints Service

- Manuscript Podcast for convenient understanding

- Global attainment for your research

- Manuscript accessibility in different formats ( Pdf, E-pub, Full Text, Audio)

- Unceasing customer service

Track the below URL for one-step submission https://juniperpublishers.com/online-submission.php 